\title{
Correction to: Joint Reliability-Aware and Cost Efficient Path Allocation and VNF Placement using Sharing Scheme
}

\author{
Abolfazl Ghazizadeh ${ }^{2} \cdot$ Behzad Akbari $^{2} \cdot$ Mohammad M. Tajiki $^{1}$ \\ Published online: 20 January 2022 \\ (c) The Author(s), under exclusive licence to Springer Science+Business Media, LLC, part of Springer Nature \\ 2022
}

\section{Correction to: Journal of Network and Systems Management (2022) 30:5 https://doi.org/10.1007/s10922-021-09613-9}

In this article the title was incorrectly given as 'Joint Reliability-Aware and Cost Efficient Path AllocationFig and VNF Placement using Sharing Scheme' but should have been 'Joint Reliability-Aware and Cost Efficient Path Allocation and VNF Placement using Sharing Scheme'.

The original article has been corrected.

Publisher's Note Springer Nature remains neutral with regard to jurisdictional claims in published maps and institutional affiliations.

The original article can be found online at https://doi.org/10.1007/s10922-021-09613-9.

Abolfazl Ghazizadeh

abolfazlghazizadeh@modares.ac.ir

Behzad Akbari

b.akbari@modares.ac.ir

Mohammad M. Tajiki

m.tajiki@qmul.ac.uk

1 School of Electronic Engineering and Computer Science, Queen Mary University of London, London, UK

2 Department of Electrical and Computer Engineering, Tarbiat Modares University, Tehran, Iran 